\title{
Isolation, Purification and Structural Characterization of a Polysaccharide from Rheum Palmatum L.
}

\author{
Jinhua Li, Sihao Zheng, Weiguang Ren, Labin Wu, Linfang Huang* \\ Institute of Medicinal Plant Development, Chinese Academy of Medical Sciences \& Peking Union Medical College, Beijing, China
}

Email address:

lfhuang@implad.ac.cn (Linfang Huang)

${ }^{*}$ Corresponding author

\section{To cite this article:}

Jinhua Li, Sihao Zheng, Weiguang Ren, Labin Wu, Linfang Huang. Isolation, Purification and Structural Characterization of a Polysaccharide from Rheum Palmatum L. Journal of Food and Nutrition Sciences. Vol. 5, No. 3, 2017, pp. 73-78. doi: $10.11648 /$ j.jfns.20170503.13

Received: February 14, 2017; Accepted: March 22, 2017; Published: April 14, 2017

\begin{abstract}
Rhubarb is a widely used Traditional Chinese Medicine in treating various diseases for thousands of years, and polysaccharide is one of the bioactive constituents in this herb. However, study of the polysaccharide is very limited, especially structural characterization. In the present study, a polysaccharide from Rheum palmatum L. (PR) was isolated and puried. Based on UV, IR, GC, GC-MS, 1H NMR and 13C NMR analyses, the structure of PR was characterized. Its molecular weight was measured to be $1.21 \times 10^{4} \mathrm{Da}$. The results of PR structural analysis showed that four $1 \rightarrow 4$ galactose were linked with one $1 \rightarrow 3$, 6 galactose and one $1 \rightarrow 6$ glucose, and each repeating unit had a branched chain consisting of a $\alpha-1 \rightarrow$ xylose, which was linked with the C-6 of galactose backbone chain.
\end{abstract}

Keywords: Rheum Palmatum L., Polysaccharide, Isolation, Purification, Structural Characterization

\section{Introduction}

Rhubarb (Da-Huang in Chinese medicine) is one of the most important herbal medicines that has been widely used for thousands of years in many Asian countries $[1,2]$. The dried roots and rhizomes of Rheum palmatum, $R$. tanguticum and $R$. offcinale are officially recorded by the Chinese Pharmacopoeia and European community monograph [1]. According to the principles of traditional Chinese medicine, rhubarb is frequently used in clinical treatment for its wide range of therapeutic activities, such as analgesic, choleretic, purgative, antibacterial, antiplatelet, anti-cancer, anti-inflammatory, antioxidant, liver-protective activities [3-5]. These pharmacological effects are attributed to components isolated from rhubarb, including anthraquinone derivatives, anthocyanin, polyphenols, polysaccharide and stilbenes $[2,6,7]$. To the best of our knowledge, secondary metabolites in the rhubarb have been widely studied by numerous researchers $[6,8]$. However, there are few reports on the structure and conformation of polysaccharides.

Polysaccharides are macromolecular carbohydrates formed from long chains of monosaccharides and their main functions include energy storage, structural support, antigens, and so on [9] Recently, extensive attention has been paid to polysaccharides due to their crucial physiological and biological functions, such as anti-coagulant, cardiac protection, immunomodulatory and antitumor $[10,11]$. According to previous reports, the biological activities of polysaccharides were closely related to their chain conformation and structure including chemical compositions, molecular weight, backbone and degree of branching [12]. In this regard, the elucidation of structure and conformation is necessary to better understand biological functions of polysaccharides. Herein. A polysaccharide from R. Palmatum (RP) was isolated and purified, and its structural characteristics were analyzed comprehensively by a variety of analytical techniques such as UV, IR, GC, GC-MS and NMR.

\section{Materials and Methods}

\subsection{Plant Materials}

R. palmatum was collected in November 2012 from Gansu Province, China. It was identified by Prof Linfang Huang. The specimen was deposited at the Herbarium of Medicinal Plant 
Development, Chinese Academy of Medical Sciences. Standards glucose (Glc), galactose (Gal) and xylose (Xyl) with $>98 \%$ purity were purchased from Sigma Chemical Co. (St. Louis, MO, USA).

\subsection{Extraction of Crude Polysaccharide}

Crude powder of $R$. palmatum was weighed for $300 \mathrm{~g}$ accurately, and added $1 \mathrm{~L}$ petroleum ether-absolute ethyl alcohol (1:1 for volume) to reflux degreasing for $2 \mathrm{~h}$. Volatile petroleum ether was removed from the residue, and added $1 \mathrm{~L}$ water to reflux extracted 3 times for each time with $2 \mathrm{~h}$. The extracting solutions were combined and concentrated to 100 $\mathrm{mL}$. The concentration was added ethanol to ethanol containing of $80 \%$, and put at $4^{\circ} \mathrm{C}$ for $12 \mathrm{~h}$, and centrifuged to obtain the precipitate. The precipitate was washed with diethyl ether and acetone several times respectively, and dried in low temperature to obtain the crude RP. The obtained crude polysaccharide was dissolved by water and added $3 \%$ papain, and put at $60^{\circ} \mathrm{C}$ for $3 \mathrm{~h}$. The solution was removed proteins by Sevag method.

\subsection{Purification of Polysaccharide}

The crude polysaccharide was re-dissolved with $5 \mathrm{~mL}$ water, and purified by $\mathrm{AB}-8(4 \mathrm{~cm} \times 80 \mathrm{~cm})$ macroporous resin column, which eluted by water with flow rate of $2 \mathrm{ml} / \mathrm{min}$, collected and concentrated eluant. The eluant was further purified by SephadexG-100 gel filtration chromatography (4 $\mathrm{cm} \times 80 \mathrm{~cm}$ ), eluted by water with flow rate of $2 \mathrm{~mL} / \mathrm{min}$. The eluant was collected by tubes respectively ( $5 \mathrm{~mL}$ per tube), and measured the absorbance by phenol-sulfuric acid method at $490 \mathrm{~nm}$. Combining the eluant, concentrating and freeze-dried were performed to obtain RP.

\subsection{Measurement of Molecular Weights}

The Polysaccharide was dissolved into the concentration of $2.0 \mathrm{mg} / \mathrm{mL}$ by water, and then filtrated by millipore filter and measured on a GPC using a Waters 1525 instrument. The purity and relative molecular weight of RP were determined on HPLC by using Waters series (Waters Ultrahydrogel TM 1000 column; ELSD detector; the temperature of column was $30^{\circ} \mathrm{C}$; the mobile phase was $0.05 \%$ mixed solution of phosphoric acid-water; isocratic elution; injection volume was $20 \mu \mathrm{L}$; flow rate was $0.4 \mathrm{~mL} / \mathrm{min}$ ). Dextran T-12.6, T-60, T-110, T-300, T-2000 and glucose were as standard [13].

\section{5. $\mathrm{NaIO}_{4}$ Oxidation}

The polysaccharide $(25.0 \mathrm{mg})$ was dissolved in $25 \mathrm{~mL} 15$ $\mathrm{mM} \mathrm{NaIO}$ solution and reacted in the dark condition. The solution was sampled every $6 \mathrm{~h}$ and followed by spectroscopy at $223 \mathrm{~nm}$. Ethylene glycol was added to terminate the reaction when the consumption of $\mathrm{IO}_{4}^{-}$was stable. Consumption of $\mathrm{IO}_{4}^{-}$was calculated according to the standard curve that was made with $\mathrm{NaIO}_{4}$ solution at $223 \mathrm{~nm}$. The content of formic acid was determined by titration using 0.01 $\mathrm{mol} / \mathrm{L} \mathrm{NaOH}$ solution.

\subsection{Monosaccharide Composition Analysis}

$50.0 \mathrm{mg}$ polysaccharide was dissolved in $4.0 \mathrm{~mL} 2 \mathrm{~mol} / \mathrm{L}$ trifluoroacetic acid (TFA) in a $10 \mathrm{~mL}$ test tube. The test tube was heated in water-bath at $100^{\circ} \mathrm{C}$ for $3 \mathrm{~h}$ to hydrolyze the polysaccharide to monosaccharides. The hydrolyzed solution was cooled to room temperature, and centrifuged for $8 \mathrm{~min}$ in $6000 \mathrm{r} / \mathrm{min}$. The supernatant was collected and evaporated to dry under reduced pressure. The dried RP was dissolved in $600 \mu \mathrm{L}$ hydroxylamine hydrochloride solution and then mixed by supersonic method for $1 \mathrm{~min}$. The mixed solution was used to carry on the water bath for at $90^{\circ} \mathrm{C} 30 \mathrm{~min}$, and cooled to room temperature. Then, $600 \mu \mathrm{L}$ acetic anhydride was added to the cooled solution to carry on water bath at $90^{\circ} \mathrm{C}$ for 30 min again, cooled to room temperature. The solution was mixed with $500 \mu \mathrm{L}$ chloroform, and next washed three times by $500 \mu \mathrm{L}$ deionized water. The organic portion was collected and removed the excess water with anhydrous $\mathrm{Na}_{2} \mathrm{SO}_{4}$. Finally, the resultant solution was evaporated to dryness and re-dissolved in $500 \mu \mathrm{L}$ chloroform for $\mathrm{GC}$ analysis. D-Glucose, D-galactose and D-xylose were also derivatized as standards. The analysis was performed using an Agilent $6890 \mathrm{~N}$ chromatograph (USA) equipped with a capillary column of DB-5 $(30 \mathrm{~m} \times 0.25 \mathrm{~mm} \times 0.25 \mu \mathrm{m})$; The GC was run using the following conditions: injection temperature, $270^{\circ} \mathrm{C}$; detector, flame-ionization detector; the temperature of detector, $270^{\circ} \mathrm{C}$; the carrier gas, $\mathrm{N} 2$ at a flow rate $30 \mathrm{~mL} / \mathrm{min}$; sample injection was as the splitless method; the temperature program was that initial temperature was $150^{\circ} \mathrm{C}$ for $3 \mathrm{~min}$, and as the rate of $3^{\circ} \mathrm{C} / \mathrm{min}$ to $170^{\circ} \mathrm{C}$, and holding for $5 \mathrm{~min}$, and then as the rate of $5^{\circ} \mathrm{C} / \mathrm{min}$ to $210^{\circ} \mathrm{C}$, and holding for $5 \mathrm{~min}$, and then as the rate of $10^{\circ} \mathrm{C} / \mathrm{min}$ to $270^{\circ} \mathrm{C}$, and holding for 5 $\min$.

\subsection{Methylation Analysis}

Methylation analysis was carried out based on the previous method [14]. $10 \mathrm{mg}$ RP which was sufficiently dried by $\mathrm{P}_{2} \mathrm{O}_{5}$ was depolymerized the methylated products and hydrolyzed by trifluoroacetic acid, and then acetylated to generate alditol acetate correspondingly. For speculating the branch structure, RP was further analyzed by partial acid hydrolysis method. RP was hydrolyzed by $0.05,0.2$ and $0.5 \mathrm{~mol} / \mathrm{L}$ trifluoroacetic acid respectively, and evaporated to dry under reduced pressure. RP was transferred to a dialysis bag after removed the trifluoroacetic acid, and dialyzed by ultrapure water for $48 \mathrm{~h}$ to obtain the high molecular weight retentate RP-a and low molecular weight permeate RP-b respectively. The solution was dried and treated with methylation process, and then analyzed by GC-MS. GC/MS analyses were conducted using a Shimadzu GC/MS QP2000 system under the same GC conditions. An electron impact quadropolar system was used in the detection.

\subsection{Infrared Spectra}

$2 \mathrm{mg}$ polysaccharide was processed by pressed-disk technique for Infrared spectra analysis A Fourier transform infrared spectropho-tometer (PerkinElmer Spectrum 400 
FT-IR, Massachusetts, USA) in the range of $4000-500 \mathrm{~cm}^{-1}$ were applied [15].

\subsection{NMR Spectra}

$12 \mathrm{mg} \mathrm{RP}$ was dissolved in $400 \mu \mathrm{L} \mathrm{D}_{2} \mathrm{O}$ for the analysis of NMR. The spectrogram of ${ }^{1} \mathrm{H}$ NMR and ${ }^{13} \mathrm{C}$ NMR were obtained with the following condition. ${ }^{1} \mathrm{H}$ NMR analysis, the frequency, $600 \mathrm{MHz}$; the temperature, $25^{\circ} \mathrm{C}$. $13 \mathrm{C} \mathrm{NMR}$ analysis, the frequency, $125 \mathrm{MHz}$; the temperature, $30^{\circ} \mathrm{C}$. TMS was used as the internal standard.

\section{Results and Discussion}

\subsection{Isolation and Purification of RP}

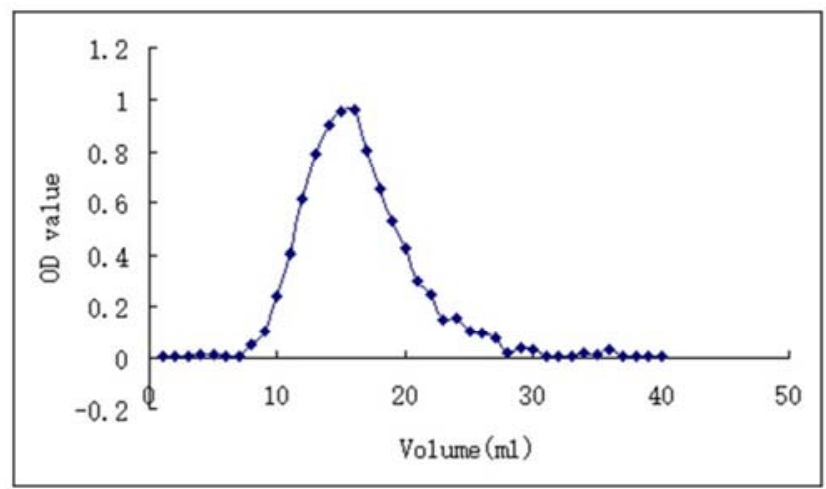

Figure 1. Size-exclusion chromatography of RP on a Sephadex G-100 column eluted with water.

Crude polysaccharide $(31.25 \mathrm{~g})$ was extracted from crude powder of $R$. palmatum by water-extraction and alcohol-sedimentation method, and then crude polysaccharide was further purified by AB-8 macroporous resin and Sephadex-G 100 column chromatography. As a result, $1.05 \mathrm{~g}$ pure polysaccharide (RP) was obtained. The absorbance was measured at $490 \mathrm{~nm}$ by phenol-sulfuric acid method. The elution curves were shown in Figure 1.

\subsection{Molecular Weight Determination}

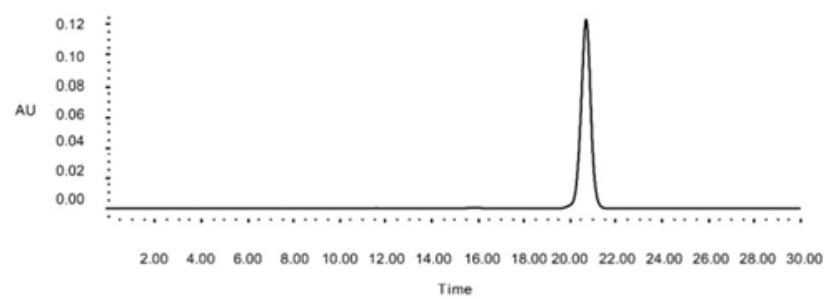

Figure 2. GPC chromatogram of RP on an Ultrahydrogel 1000 column (7.8 $\mathrm{mm} \times 300 \mathrm{~mm}$ ).

As showed in Figure 2, GPC chromatogram showed that the chromatographic peak of RP was single symmetrical peak of a homogeneous component. Based on the standard curve which was calculated by dextran $(\mathrm{Y}=12.171-0.4476 \mathrm{X}, \mathrm{r}=0.9996)$ and the retention time of samples, the relative molecular weight of $\mathrm{RP}$ was determined to be $1.21 \times 10^{4} \mathrm{Da}$. The UV scanning in $260 \mathrm{~nm}$ and $280 \mathrm{~nm}$ wavelength shown that RP had no absorption, and the ninhydrin reaction was negative, which indicated that RP did not contain nucleic acid and protein.

\section{3. $\mathrm{NaIO}_{4}$ Oxidation Reaction}

The glycosidic linkage locations of RP were preliminarily determined by consumption of $\mathrm{NaIO} 4$ and production of formic acid. The results showed that $1 \mathrm{~mol}$ of sugar residues consumed $0.64 \mathrm{~mol}$ of $\mathrm{NaIO} 4$ and produced $0.08 \mathrm{~mol}$ of formic acid. Meanwhile, the consumption of $\mathrm{NaIO}_{4}$ was two times higher than the production of formic acid, which indicated that RP contained linkages which could produce formic acid such as $(1 \rightarrow 6,1 \rightarrow)$ linkage, but also contained some amount of linkage types which only could consume NaIO4 such as $(1 \rightarrow 4)$ linkage or some amount of linkage types which could not be oxidized by $\mathrm{NaIO}_{4}$ such as $(1 \rightarrow 3,6)$ linkage.

\subsection{Monosaccharide Composition Analysis}

In order to obtain more information about the monosaccharide residues of $\mathrm{RP}$, the product of $\mathrm{NaIO}_{4}$ oxidation was degraded by Smith degradation method and then analyzed by GC. The chromatograms of standard monosaccharides and degradation products were shown in Figure 3. The results indicated that RP was mainly consist of galactose as well as a small amount of glucose and xylose, whose molar ratio was 5.1: 1: 1 .
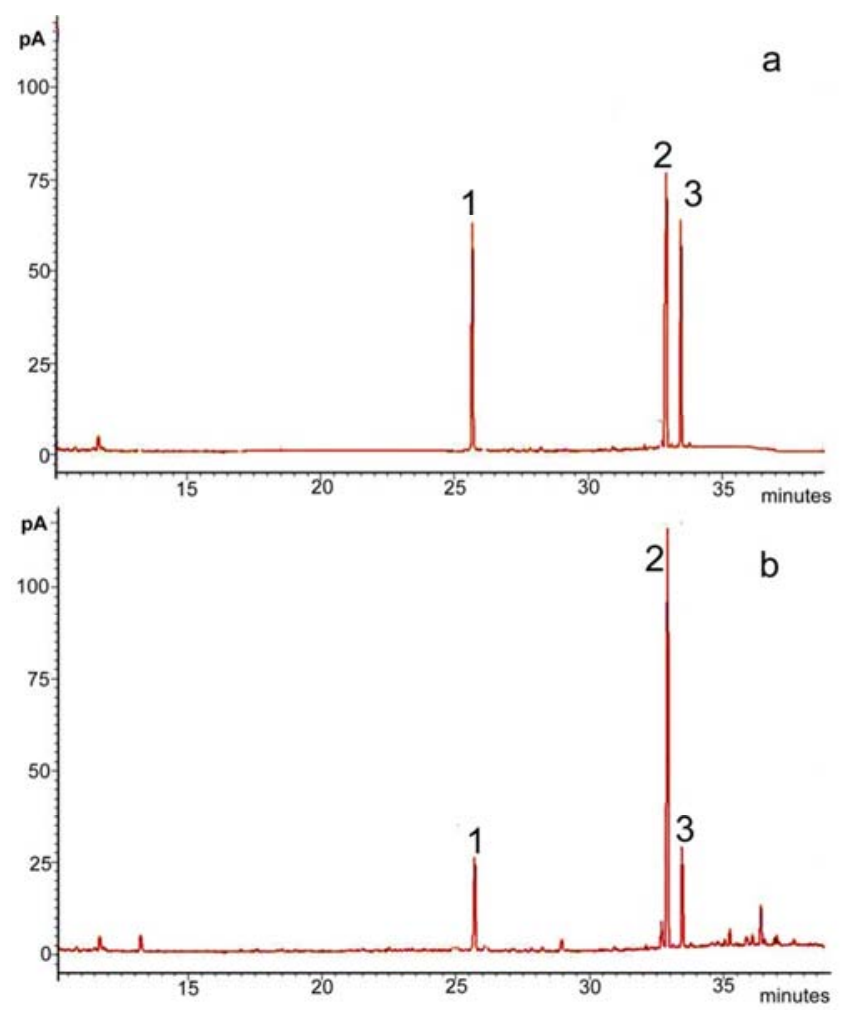

Figure 3. GC analysis of acetylated standard monosaccharide (a) and acetylated RP (b), 1. Xylose 2. Galactose 3. Glucose.

\subsection{Methylation Reaction}

To further determine the linkage types between 
monosaccharide residues, RP was permethylated, hydrolyzed and converted into partially methylated alditol acetates for GC-MS analysis. The results were shown in Table 1, the backbone structure of the sugar chain was mainly consist of $1 \rightarrow 4$ linked galactose $(\mathrm{Gal}), 1 \rightarrow$ terminal xylosel $(\mathrm{Xyl})$ and $1 \rightarrow 3,6$ linked galactose (Gal), small amounts of $1 \rightarrow 6$ linked glucose (Gal), $1 \rightarrow 3$ linked glucose (Glc) and galactose (Gal). In Mass spectrum, m/z 43 was the base peak of methylated acid sugar esters (PMAA), and was formed due to the loss of acetyl ion $(\mathrm{CH} 3 \mathrm{CO}+)$. The $\mathrm{m} / \mathrm{z} 145$ and 259 fragments were the characteristic peaks of hexose mass spectrum, while the erythritol and glycerol were generated.

Table 1. Methylation analysis of RP.

\begin{tabular}{|c|c|c|c|}
\hline Methylated alditol & Mainion ion fragment $(\mathrm{m} / \mathrm{z})$ & Type of linkage & Molar ratio \\
\hline 2,4,6--tri-O-methyl galactitol & 434587101117129161259 & $\rightarrow 3) \mathrm{Gal} \rightarrow 1$ & 3.0 \\
\hline 2,3,6--tri-O-methyl galactitol & 43458799101113117161233 & $\rightarrow 4) \mathrm{Gal} \rightarrow 1$ & 41.0 \\
\hline 2,4--tri-O-methyl galactitol & $437385117,129,189,233$ & $\rightarrow 3,6) \mathrm{G}$ alp $\rightarrow 1$ & 15.8 \\
\hline 2,4,6-tri-O-methyl glucitol & 434587101117129161 & $\rightarrow 3) \mathrm{Glc} \rightarrow 1$ & 3.6 \\
\hline 2,3,4-tri-O-methyl glucitol & 438799101117129189 & $\rightarrow 6)$ Glc $\rightarrow 1$ & 10.4 \\
\hline 2,3--tri-O-methyl Xyl & 437187101117129143161 & $\mathrm{Xyl} \rightarrow 1$ & 26.2 \\
\hline
\end{tabular}

The further research on branch structure of RP was shown in Table 2. After partial acid hydrolysis, the macromolecular fragment RP-a was the RP backbone structural components which consisted of galactose and glucose. Thus, the backbone structure of RP was composed of galactose and glucose alternately. The analysis by GC-MS for RP-b was shown to be $1 \rightarrow$ terminal xylose. Because the RP-b was the lower molecular weight part with partial acid hydrolysis, so it was hydrolyzed as a branched chain of RP.

Table 2. Methylation analysis of $R P-a$ and $R P-b$.

\begin{tabular}{lllll}
\hline Group & Methylated alditol & Mainion ion fragment $\mathbf{( m / z )}$ & Type of linkage & Molar ratio \\
\hline \multirow{4}{*}{ RP-a } & $2,4,6$--tri-O-methyl galactitol & 434587101117129161 & $\rightarrow 3) \mathrm{Gal} \rightarrow 1$ & 4.3 \\
& $2,3,6-$-tri-O-methyl galactitol & 43458799101113117161233 & $\rightarrow 4) \mathrm{Gal} \rightarrow 1$ & 58.4 \\
& $2,4-$-tri-O-methyl galactitol & 437385117133147159 & $\rightarrow 3,6) \mathrm{G} \mathrm{al} \rightarrow 1$ & 22.5 \\
RP-b & 2,3,4-tri-O-methyl glucitol & 438799101117129189 & $\rightarrow 6) \mathrm{Glc} \rightarrow 1$ & 14.8 \\
\hline
\end{tabular}

\subsection{Infrared Spectroscopy Analysis}

To characterize polysaccharide fractions, the characteristic absorption of RP was performed in the range of 4000-400 $\mathrm{cm}^{-1}$ by IR. The result was shown in Figure 4 . It is indicated that distinct polysaccharide characteristic bands appeared at 3435 , $2951,1648,1617,1413$ and $1101 \mathrm{~cm}^{-1}$. The wide and strong absorption band at $3435 \mathrm{~cm}^{-1}$ was the $\mathrm{O}-\mathrm{H}$ stretching vibration of sugar; resulting from intermolecular and intramolecular hydrogen bonding [16]. A weak peak at $2951 \mathrm{~cm}^{-1}$ was caused by $\mathrm{C}-\mathrm{H}$ asymmetric stretching vibration; The peak at 1617 cm-1 was caused by the H-O-H bending of water adsorbed on the polysaccharide sample; The peak near $1413 \mathrm{~cm}-1$ was caused by the vicinity of deformation vibration of $\mathrm{CH}_{2}$; the peak near $1101 \mathrm{~cm}^{-1} 1$ was attributed to the stretching vibration of C-O-C and the deformation vibration of $\mathrm{O}-\mathrm{H}$ in $\mathrm{C}-\mathrm{O}-\mathrm{H}$ bond [17]. $955 \mathrm{~cm}^{-1}$ was caused by the symmetric stretching vibration of the furan ring; $779 \mathrm{~cm}^{-1}$ was caused by the stretching vibration of the pyran ring. Hence, monosaccharide residues in RP was appeared in form of furan ring and pyran ring. The result is consistent with monosaccharide composition analysis.

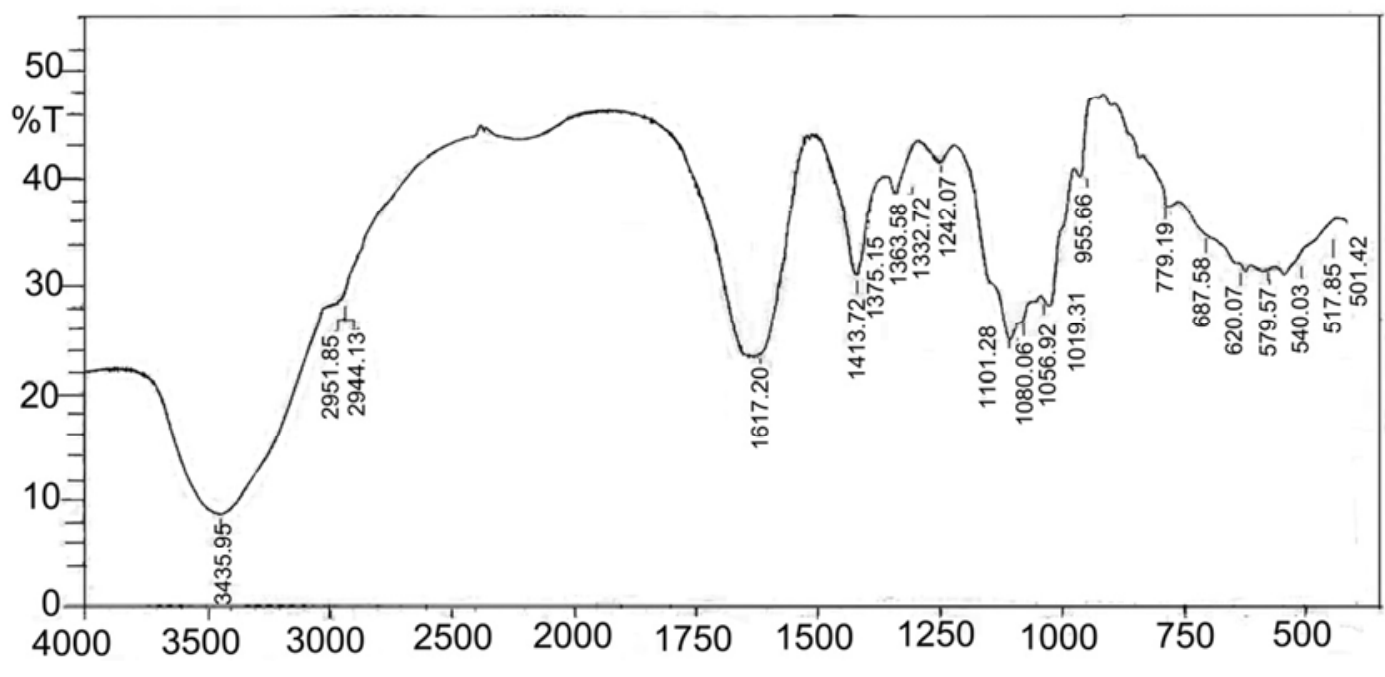

Figure 4. The FT-IR spectrum of RP. 


\subsection{NMR Analysis}

The structural feature of RP was also elucidated by NMR spectral analysis. The spectrums were shown in Figure 5. The chemical shifts at $\delta 4.30-5.90 \mathrm{ppm}$ in the ${ }^{1} \mathrm{HNMR}$ spectrum could be assigned to the typical signals of anomeric protons. In the spectrum, three main signals at $\delta 5.47,5.14$ and 4.47 in the anomeric proton region indicated that RP was mainly composed of three types of monosaccharides. Among them, one signal at $\delta 4.47$ was assigned to the configuration of $\beta$-glycosidic bond and others were assigned to the configuration of $\alpha$-glycosidic bond. The strong signal at around $4.8 \mathrm{ppm}$ was attributed to the chemical shifts of $\mathrm{D}_{2} \mathrm{O}$.
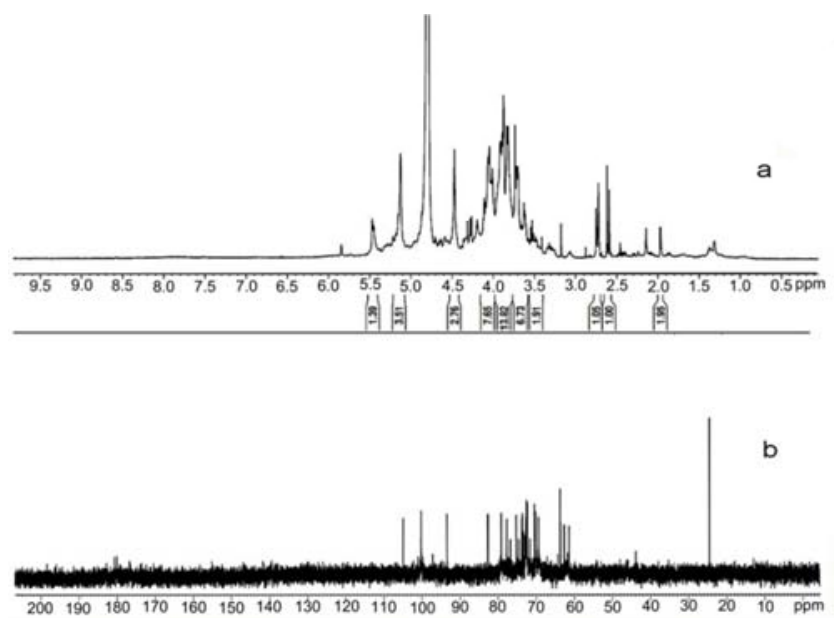

Figure 5. The ${ }^{1} H$ NMR spectrum (a) and ${ }^{13} C N M R$ spectrum (b) of RP.

Correspondingly the ${ }^{13} \mathrm{C}$ NMR spectrum showed three carbon signals of $\delta 104.966,100.244$ and 93.454 in anomeric region, which were assigned to $\beta$-xylose, $\alpha$-galactose and $\alpha$-glucose, respectively. This is consistent with those obtained in GC and GC-MS analysis. In the non-anomeric carbon region, a large number of signals accumulated in the range of $\delta$ $61 \sim 77$. The absorption peak in $\delta 82.6$ indicated that the substitution occurred on $\mathrm{C} 3$ of glucose; the appears of signal $\delta 79.19$ indicated that substitution occurred on $\mathrm{C} 4$ of galactose residues; the strong absorption peak of signal $\delta 82.6$ indicated that the substitution occurred on $\mathrm{C} 3$ of glucose residue; the presence of signal $\delta 63.6$ indicated that sugar chain contained fructofuranosyl units; the strong absorption peak at $\delta 62.6$ indicated that $\mathrm{C} 6$ was not substituted in most glucose.

The combined results from the analysis of NaIO4 oxidation, Smith degradation, IR, GC-MS and NMR indicated that the backbone chain of RP was composed of galactose linked by $1 \rightarrow 4$ and $1 \rightarrow 3,6$ glycosidic bonds as well as glucose linked by $1 \rightarrow 6$ glycosidic bonds, and the branch chains were attached to backbone chain by xylose linked by $1 \rightarrow$ terminal glycosidic bonds. Namely, four $1 \rightarrow 4$ galactose were linked with one $1 \rightarrow$ 3,6 galactose and one $1 \rightarrow 6$ glucose, and each repeating unit had a branched chain consisting of a $\alpha-1 \rightarrow$ xylose, which was linked with the C- 6 of galactose backbone chain.

\section{Conclusion}

In conclusion, a polysaccharide from R. Palmatum (RP) was obtained in this study. The relative molecular weight of RP was $1.21 \times 10^{4}$. Through structural characterization analysis, it is demonstrated that the backbone chain of RP is composed of glucose linked by $\alpha-(1 \rightarrow 4)$ galactose, $\alpha-(1 \rightarrow 3,6)$ galactose and $\alpha-(1 \rightarrow 6)$ glucose, the branch chains are attached to backbone chain by $\beta-1 \rightarrow$ terminal xylose. RP was built up of $\beta$-xylose, $\alpha$-galactose and $\alpha$-glucose residues in the molar ratio of 1:5.1:1. This study provided useful information for further study of the polysaccharide from $R$. palmatum.

\section{Acknowledgements}

This study was supported by the National Natural Science Foundation of China (No. 81274013 and 81473315), Key National Natural Science Foundation of China (No. 81130069). All the author did not have any potential conflict of interest.

\section{References}

[1] Sun, M.; Li, L.; Wang, M.; van Wijk, E.; He, M.; van Wijk, R.; Koval, S.; Hankemeier, T.; van der Greef, J.; Wei, S., Effects of growth altitude on chemical constituents and delayed luminescence properties in medicinal rhubarb. Journal of photochemistry and photobiology. B, Biology 2016; 162: 24-33.

[2] Ren;, G.; Li;, L.; Hu;, H.; Li;, Y.; Liu;, C.; Wei, S., Influence of the Environmental Factors on the Accumulation of the Bioactive Ingredients in Chinese Rhubarb Products. 2016;11(5): e0154649.

[3] Sun, W.; Zhang, X.; Zhang, Z.; Zhu, R., Data fusion of near-infrared and mid-infrared spectra for identification of rhubarb. Spectrochimica acta. Part A, Molecular and biomolecular spectroscopy 2017; 171: 72-79.

[4] Zhu, T.; Liu, X.; Wang, X.; Cao, G.; Qin, K.; Pei, K.; Zhu, H.; Cai, H.; Niu, M.; Cai, B., Profiling and analysis of multiple compounds in rhubarb decoction after processing by wine steaming using UHPLC-Q-TOF-MS coupled with multiple statistical strategies. Journal of separation science 2016; 39 (15): 3081-90.

[5] Lin, T. J.; Lin, C. F.; Chiu, C. H.; Lee, M. C.; Horng, J. T., Inhibition of endosomal fusion activity of influenza virus by Rheum tanguticum (da-huang). Scientific reports 2016; 6: 27768.

[6] Liu, Y.; Li, L.; Xiao, Y. Q.; Yao, J. Q.; Li, P. Y.; Yu, D. R.; Ma, Y. L., Global metabolite profiling and diagnostic ion filtering strategy by LC-QTOF MS for rapid identification of raw and processed pieces of Rheum palmatum L. Food chemistry 2016; 192: 531-40.

[7] Takeoka, G. R.; Dao, L.; Harden, L.; Pantoja, A.; Kuhl, J. C., Antioxidant activity, phenolic and anthocyanin contents of various rhubarb (Rheumspp.) varieties. International Journal of Food Science \& Technology 2013; 48 (1): 172-178. 
[8] Zheng, Q. X.; Hai-Feng, W. U.; Jian, G.; Nan, H. J.; Chen, S. L.; Yang, J. S.; Xu-Dong, X. U., Review of Rhubarbs: Chemistry and Pharmacology. Chinese Herbal Medicines 2013; 5 (1): $9-32$.

[9] Tang, J.; Nie, J.; Li, D.; Zhu, W.; Zhang, S.; Ma, F.; Sun, Q.; Song, J.; Zheng, Y.; Chen, P., Characterization and antioxidant activities of degraded polysaccharides from Poria cocos sclerotium. Carbohydrate polymers 2014; 105: 121-6.

[10] Zhang, X.; Liu, L.; Lin, C., Isolation, structural characterization and antioxidant activity of a neutral polysaccharide from Sisal waste. Food Hydrocolloids 2014; 39 : 10-18.

[11] Hu, H.; Liang, H.; Wu, Y., Isolation, purification and structural characterization of polysaccharide from Acanthopanax brachypus. Carbohydrate polymers 2015; 127: 94-100.

[12] Ye, H.; Zhou, C.; Li, W.; Hu, B.; Wang, X.; Zeng, X., Structural elucidation of polysaccharide fractions from brown seaweed Sargassum pallidum. Carbohydrate polymers 2013; 97 (2): 659-64.

[13] Wen, L.; Lin, L.; You, L.; Yang, B.; Jiang, G.; Zhao, M., Ultrasound-assited extraction and structural identification of polysaccharides from Isodon lophanthoides var. gerardianus (Bentham) H. Hara. Carbohydrate polymers 2011; 85 (3): 541-547.

[14] Anumula, K. R.; Taylor, P. B., A comprehensive procedure for preparation of partially methylated alditol acetates from glycoprotein carbohydrates. Analytical biochemistry 1992; 203 (1): 101 .

[15] Wang, X. T.; Zhu, Z. Y.; Zhao, L.; Sun, H. Q.; Meng, M.; Zhang, J. Y.; Zhang, Y. M., Structural characterization and inhibition on alpha-d-glucosidase activity of non-starch polysaccharides from Fagopyrum tartaricum. Carbohydrate polymers 2016; 153: 679-85.

[16] Nep, E. I.; Carnachan, S. M.; Ngwuluka, N. C.; Kontogiorgos, V.; Morris, G. A.; Sims, I. M.; Smith, A. M., Structural characterisation and rheological properties of a polysaccharide from sesame leaves (Sesamum radiatum Schumach. \& Thonn.). Carbohydrate polymers 2016; 152: 541-7.

[17] He, P. F.; He, L.; Zhang, A. Q.; Wang, X. L.; Qu, L.; Sun, P. L., Structure and chain conformation of a neutral polysaccharide from sclerotia of Polyporus umbellatus. Carbohydrate polymers 2017; 155: 61-67. 